\title{
Micro-machining workstation for a diode pumped Nd:YAG high-brightness laser system
}

\author{
R. A. Kleijhorst and H. L. Offerhaus \\ NCLR BV, Postbus 2662, 7500 CR Enschede, The Netherlands \\ P. Bant \\ ILT BV, Tinsteden 30, 7547 TG Enschede, The Netherlands
}

(Received 5 November 1997; accepted for publication 4 February 1998)

\begin{abstract}
A Nd:YAG micro-machining workstation that allows cutting on a scale of a few microns has been developed and operated. The system incorporates a telescope viewing system that allows control during the work and a software interface to translate AutoCad files. Some examples of the performance are given. With this setup we demonstrate the possibility of machining within a few microns with a Nd:YAG laser. (C) 1998 American Institute of Physics. [S0034-6748(98)02405-8]
\end{abstract}

\section{INTRODUCTION}

The field of laser materials processing covers processing with a wide variety of lasers. In general, there is a trade-off between power and beam quality which implies that high power leads to relatively big spotsizes. Since the spotsize also scales with the wavelength, a smaller focus can be achieved by a laser with a shorter wavelength. For machining at the scale of a few microns, excimer lasers are used. In these lasers the small wavelength allows for the machining of small structures despite the low beam quality. For solidstate lasers pumping with diode lasers is much more efficient than traditional flashlamp pumping. Therefore higher average loading levels are now possible without loss of quality. Other advantages are the stability, reliability and increased lifetime. With diode pumping and careful thermal management it is therefore possible to use a Nd:YAG laser for materials processing with a precision of a few microns. The laser system developed by Nederlands Centrum voor Laser Research (NCLR) is specified at $1 \mathrm{kHz}$ repetition rate, $10 \mathrm{~ns}$ Q-switched pulse or $160 \mu \mathrm{s}$ free running pulse, $5 \mathrm{~mJ} /$ pulse and a transverse electromagnetic $(\mathrm{TEM})_{00}$ mode $\left(M^{2}<1.06\right)$. The workstation is designed for a $2 \mu \mathrm{m}$ (diffraction limited) focus diameter, $0.1 \mu \mathrm{m}$ accuracy and reproducible positioning, feedback and control during the processing and user friendly software control. Commercially available systems such as the LAY $50 \mathrm{~K}$ by Haas Lasers, ${ }^{1}$ the KLS 246 by Lasag ${ }^{2}$ and the RSY 150 P2 by Rofin Sinar ${ }^{3}$ cannot focus below 20 $\mu \mathrm{m}$, due to multimode operation and focusing with a low numerical aperture. They are therefore not suited for micromachining with a spotsize below $20 \mu \mathrm{m}$. A smaller spotsize can be achieved by the introduction of an aperture but this reduces the output power considerably. Our system uses the full area of the laser beam. The recently announced QUICKLAZE by New Wave Research ${ }^{4}$ achieves a resolution of $1 \mu \mathrm{m}$ at the third harmonic of $1064 \mathrm{~nm}$ but has a repetition rate of only $20 \mathrm{~Hz}$.

\section{DESIGN}

A detailed schematic of the "head" of the workstation is shown in Fig. 1. Figure 2 shows a schematic of the complete workstation from the side view. The head consists of three parts. The middle one is fixed to a frame and the upper and lower part are connected by three steel rods. The laser light travels through the inside of a Newport X-95 macro bench profile and is deflected downward with the laser mirror. The small focus diameter is achieved by focusing with a large numerical aperture. In order to keep a reasonable working distance, the beam diameter is first expanded 100 times (to $50 \mathrm{~mm}$ diameter), by a telescope inside the $\mathrm{X}-95$ profile. The focusing is done with a triplet that is specially designed to minimize spherical aberration at these large apertures. The focus depth is only $0.8 \mu \mathrm{m}$ due to the high numerical aperture. A differential micrometer with a resolution of $0.5 \mu \mathrm{m}$ is used for the height displacement. The control of the material processing is done with a fast charge-coupled device (CCD) camera. The design of the control optics is such that the image remains sharp when the focusing triplet is adjusted. This is achieved by placing a second lens in the upper part above the deflection mirror (which is transparent for visible light) so that it always has a fixed distance to the focusing triplet. This $1 \times$ telescope provides an image at a fixed distance to the object independent of the placement of the telescope in between. ${ }^{5}$ The image is then magnified approximately 165 times (again with a telescope) before it is recorded by the CCD camera. The magnification ratio from the surface of the CCD array to the monitor is about 20 times, so that the total magnification of the object is approximately 3300 times. This means that an object of $1 \mu \mathrm{m}$ is seen as an image of $3.3 \mathrm{~mm}$ on the screen. In order to prevent the camera from being blinded by the interacting laser pulse, the camera is synchronized to the laser system so that the camera will only record images in between the interacting laser pulses. To make sure that the workstation is insensitive to vibrations, Newport X-95 profile is used to construct the frame for the optical and mechanical part of the system. Another advantage of this frame is the possibility to run the laser beam inside the profile for extra safety. Vibrations have been tested and no visible effects were detected. Other effects such as temperature variation or misalignment have been modeled. The effect of a temperature increment of 


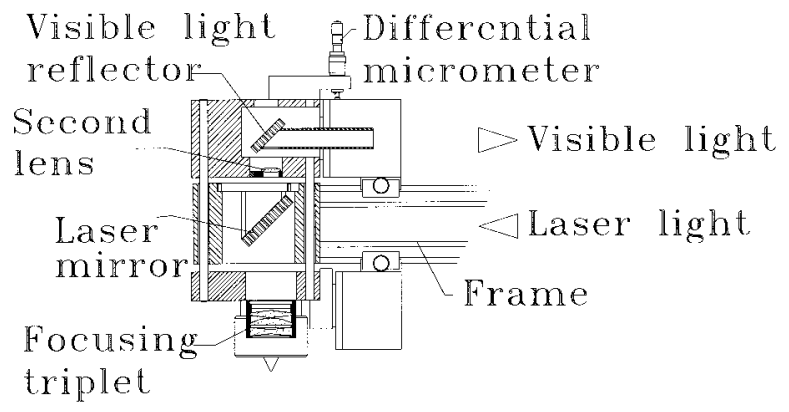

FIG. 1. Schematic of the "head" of the workstation with the focusing triplet, deflection mirror, height adjustment and control telescope.

$10{ }^{\circ} \mathrm{C}$ of the workstation results in a shift of the position of the focus of approximately $10 \mu \mathrm{m}$. However, the diameter of the focus remains at $2 \mu \mathrm{m}$.

\section{SOFTWARE}

The XY table is equipped with a controller that can handle a variety of commands to form so-called trajectories. To facilitate the usage of the workstation, a software interface was developed to handle the communication between a PC and the XY controller. The software interface was developed by Industriële Laser Toepassingen (ILT). It contains modules that take an AutoCad dxf file as input and calculate the tool path, piercing points and the tool correction. The tool correction is performed automatically, i.e., the system distinguishes between outer and inner tool paths. The user is not troubled with the choice of direction of the tool correction. A postprocessor module adds the International Standards Organization (ISO) codes to the calculated curves, together with machine specific codes, such as laser parameters, etc. The modular design of the software certifies the possibility to control different kinds of computer numerical controlled $(\mathrm{CNC})$ machines. Further work is done to prepare the software for WINDOWS-95. For commercial applications the laser can be integrated into the workstation.

\section{LASER}

The laser is a diode pumped Nd:YAG laser that is currently under development at NCLR. It is a stable ring oscil-

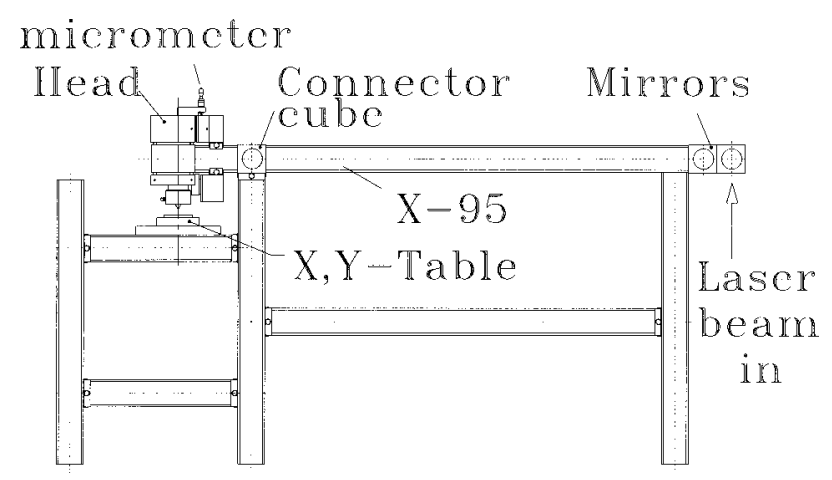

FIG. 2. Schematic of the total experimental setup (without the laser).
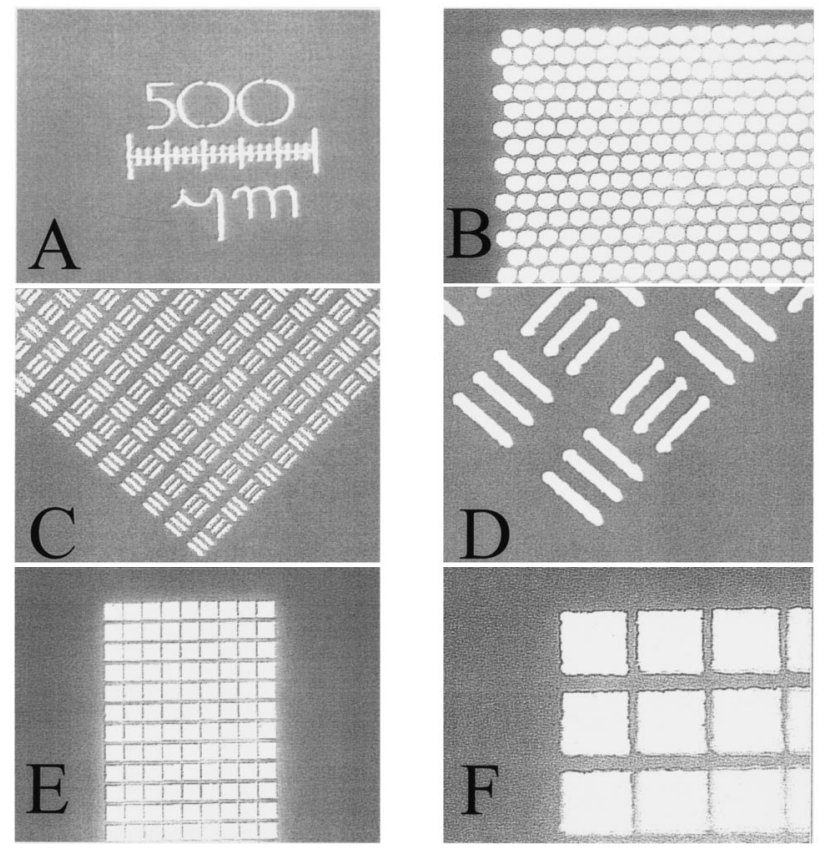

FIG. 3. Examples of the possibilities for micro-machining with Nd:YAG: (A) $500 \mu \mathrm{m}$ ruler; (B) $100 \mu \mathrm{m}$ holes for a $200 \mu \mathrm{m}$ radiation high reflector; (C)-(D) $60 \mu \mathrm{m}$ slits in a thatch pattern; (E)-(F) $100 \mu \mathrm{m}$ squares for a 200 $\mu \mathrm{m}$ radiation high reflector.

lator with a Q-switch and a zigzag-geometry miniature slab. The whole laser takes about $25 \times 15 \times 10 \mathrm{~cm}^{3}$. It is pumped with quasi-continuous diodes. The maximum repetition rate is $1 \mathrm{kHz}$. Even at these high repetition rates the $M^{2}$ factor for the beam quality remains below 1.1. Further information on this oscillator can be found in Ref. 6 .

\section{RESULTS}

Examples of small scale material processing are shown in Fig. 3. These examples show structures cut in $20 \mu \mathrm{m}$ aluminum foil. B are holes of $100 \mu \mathrm{m}$ diameter which constitute a high reflector for $200 \mu \mathrm{m}$ radiation. $\mathrm{E}$ and $\mathrm{F}$ show a similar device in squares (also $100 \mu \mathrm{m}$ wide). C and D are $60 \mu \mathrm{m}$ slits in a thatch pattern. The spotsize in the examples is about $5 \mu \mathrm{m}$. All examples were cut without the use of shielding gas. The gas flow was found to disturb the foil surface.

\footnotetext{
${ }^{1} \mathrm{H}$. Laser, Features prospectus, type LAY 50K (1993).

${ }^{2}$ Lasag, Features prospectus, type KLS 246 (1993).

${ }^{3}$ R. Sinar, Features prospectus, type RSY 150 P2 (1993).

${ }^{4}$ E. North, Laser Focus World 33, 149 (1997).

${ }^{5}$ H. L. Offerhaus and R. A. Kleijhorst, Dutch patent application no. 10.07068 (1997).

${ }^{6}$ H. P. Godfried and H. L. Offerhaus, XI International Symposium on Gas Flow and Chemical Lasers and High Power Laser Conference, edited by H. Baker, Proc. SPIE 3092 (SPIE, Bellingham, WA, 1996), pp. 29-32.
} 